\title{
KINETICS OF INDIUM ATOMIZATION FROM DIFFERENT ATOMIZER SURFACES IN ELECTROTHERMAL ATOMIC ABSORPTION SPECTROMETRY (ETAAS)
}

\author{
XIU-PING YAN and ZHE-MING $\mathrm{N}^{*}$ \\ Research Center for Eco-Environmental Sciences, Academia Sinica, P.O. Box 2871, Beijing, China 100085
}

XiaO-TAO YANG

Institute of Rock and Mineral Analysis, Beijing, China

GUO-QIANG HoNG

Factory of Geological Instrument, Beijing, China

(Received 3 December 1992. Revised 18 May 1993. Accepted 20 May 1993)

\begin{abstract}
Summary-The kinetic parameters of indium atomization in electrothermal atomic absorption spectrometry (ETAAS) have been determined by a newly proposed method. Effect of the atomizer surface and the palladium modifier on the kinetics of indium atomization has been investigated. The mechanisms of indium atomization seem to be identical for the pyrolytically coated graphite and the uncoated graphite tubes, i.e. the rate-limiting step for the atomization changes from a first order kinetics at lower temperatures into a nearly $1 / 3$ order kinetics at higher temperatures, which may suggest that the analyte moves from a dispersed state to agglomates with increasing temperature. However, for the zirconium coated graphite tube, the atomization of indium is controlled by a single mechanism with the kinetic order of near $2 / 3$ and the activation energy of $186 \pm 13 \mathrm{~kJ} / \mathrm{mol}$. Relatively weak indium-zirconium carbide interactions and the release of indium from the sphere of molten indium metal on the zirconium coated surface are suggested. In the presence of palladium, a simple mechanism, i.e. the release of indium from the solid solution of the In and the Pd on the pyrolytically coated graphite surface, is proposed to account for the observed first order kinetics and the activation energy of $421 \pm 27 \mathrm{~kJ} / \mathrm{mol}$.
\end{abstract}

The atomization of analytes in electrothermal atomic absorption spectrometry (ETAAS) has been studied by many researchers based on kinetic, $^{1-5}$ thermodynamic ${ }^{6-10}$ and combined kinetic-thermodynamic considerations. ${ }^{11-15}$ Usually, most of these reports focused on the atomization mechanisms of analytes without matrices nor modifiers from one type of graphite surface. However, it is more important to elucidate the mechanisms of analyte released in the presence of matrices or modifiers from different atomizer surfaces since normal analysis is often carried out under such conditions. Thus far, there have been a few papers appearing to deal with the atomization mechanisms of the analyte from various atomizer surfaces. Aggett and Sprott $^{16}$ compared the appearance temperatures for the atomization of various analytes

*Author for correspondence. from both graphite and tantalum surfaces, and concluded that the most possible mechanism for the analyte atomization from a tantalum surface was the thermal decomposition of the oxide. Gregoire et al. ${ }^{17}$ studied the atomization mechanisms of $\mathrm{U}, \mathrm{V}, \mathrm{Mo}, \mathrm{Ni}, \mathrm{Mn}, \mathrm{Cu}$ and $\mathrm{Mg}$ from pyrolytic graphite and tantalum surfaces. The atomization mechanisms of various analytes from molybdenum ${ }^{18}$ and tungsten ${ }^{19}$ surfaces were compared with those from the graphite surface. $\mathrm{Gao}$ and $\mathrm{Ni}^{20}$ suggested that the atomization mechanism of germanium in the zirconium coated graphite tube was the vaporization of germanium following the reduction of $\mathrm{GeO}_{2}$ or $\mathrm{GeO}$ by zirconium carbide, which was different from the thermal decomposition mechanism of the oxide in the uncoated graphite tube. Recently, Chakrabarti and Cathum ${ }^{21}$ investigated the mechanism of cobalt atomization from different atomizer surfaces in ETAAS, and suggested that the atomization mechanism of 
cobalt seemed to be the same for the pyrolytically coated graphite and the uncoated electrographite surfaces, but different for the glassy carbon furnace. Fonseca et $a .^{22}$ studied the vaporization/atomization characteristics of $\mathrm{Cu}$ sample from graphite and Ta-lined tubes using both $\mathrm{Ta}$ and graphite platforms and found first order release of $\mathrm{Cu}$ from both $\mathrm{Ta}$ and graphite surfaces.

Much less attention has been paid to the behavior of indium in the graphite furnace compared with other analytes. Grinshtein et $a l^{23}$ studied the interaction between indium and the graphite surface at various temperatures by using a tube-in-tube, spatially isothermal furnace. At temperatures less than $1900 \mathrm{~K}$, indium adsorbed to the graphite surface and the effective residence time of this element was greater than theoretically predicted residence time. However, at temperatures greater than $1900 \mathrm{~K}$, no significant adsorption of In occurred. In an earlier study, Katskov et al..$^{24}$ determined an activation energy of $234 \mathrm{~kJ} / \mathrm{mol}$ at lower temperatures followed by $134 \mathrm{~kJ} / \mathrm{mol}$ at higher temperatures for the atomization of In. Recently, McNally and Holcombe ${ }^{25}$ studied the topology and vaporization characteristics of indium in a CRA- 90 min. Massmann furnace. Two activation energies of $238 \pm 29$ and $138 \pm 21 \mathrm{~kJ} / \mathrm{mol}$ were obtained over the course of release from In using manual pipetting. An appearance order of greater than one was suggested for indium release from the concentration study, and the aggregate surface was proposed as one of the generation mechanisms for indium. They also observed the relatively weak interaction between indium and graphite.

The purpose of this work is to study the atomization kinetics of indium from different atomizer surfaces, i.e. pyrolytically coated, uncoated and zirconium coated graphite surfaces, and the effect of the palladium modifier on the kinetics of indium atomization from the pyrolytically coated graphite surface by using a newly proposed method. ${ }^{26}$

\section{THEORY}

In a previous report, ${ }^{26}$ a method was developed for the simultaneous determination of the kinetic parameters of atom formation from a single absorbance signal profile under normal analytical conditions in electrothermal atomic absorption spectrometry (ETAAS). The change of the absorbance, $A$, with time, $t$, was given by the following equation:

$\mathrm{d} A / \mathrm{d} t=P^{x-1} k_{1}\left(\int_{1}^{\infty} k_{2} A \mathrm{~d} t-A\right)^{x}-k_{2} A$

where $k_{1}$ and $k_{2}$ are the temperature and subsequently time dependent rate constants for the formation and dissipation of gaseous atoms, respectively; $P$ a proportionality factor, $x$ the kinetic order of the atom formation reaction. According to the Arrhenius' law, the temperature dependence of $k_{1}$ can be expressed as follows:

$$
k_{1}=k_{0} \cdot \exp \left(-E_{\mathrm{a}} / R T\right)
$$

where $k_{0}$ and $E_{a}$ are the frequency factor and activation energy for atom formation, respectively; $R$ the gas constant and $T$ the absolute temperature at time $t$. Substituting equation (2) into equation (1) and rearranging yields:

$$
\begin{aligned}
& \mathrm{d} A / \mathrm{d} t+k_{2} A=k_{0} P^{x-1} \\
& \quad \times\left(\int_{t}^{\infty} k_{2} A \mathrm{~d} t-A\right)^{x} \cdot \exp \left(-E_{a} / R T\right)
\end{aligned}
$$

Taking logarithm of equation (3), differentiating and rearranging give

$$
\begin{aligned}
& \Delta \ln \left(\mathrm{d} A / \mathrm{d} t+k_{2} A\right) / \Delta \ln \left(\int_{t}^{\infty} k_{2} A d t-A\right)= \\
& x-\left(E_{a} / R\right) \Delta(1 / T) / \Delta \ln \left(\int_{t}^{\infty} k_{2} A \mathrm{~d} t-A\right) .
\end{aligned}
$$

Equation (4) shows that if the atomization of the analyte from the atomizer surface is governed by a single kinetic mechanism, a plot of $\Delta \ln \left(\mathrm{d} A / \mathrm{d} t+k_{2} A\right) / \Delta \ln \left(\int_{t}^{\infty} k_{2} A \mathrm{~d} t-A\right) \quad v s$ $\Delta(1 / T) / \Delta \ln \left(\int_{t}^{\infty} k_{2} A \mathrm{~d} t-A\right)$ should be a straight line. From the slope of such a plot, the activation energy $\left(E_{\mathrm{a}}\right)$ can be calculated, while the kinetic order $(x)$ can be obtained from the intercept. The validity of equation (4) has been confirmed both by computer simulation study ${ }^{26,27}$ and the ETAAS experiments. ${ }^{26}$

To obtain the kinetic order and activation energy by using equation (4), the temperature dependence of $k_{2}$ should be known. In this study, the gas-stop mode is used in the atomization step and the dissipation of the analyte by convection is distinctly negligible. Therefore, $k_{2}$ is the total rate constant for the dissipation of the analyte by diffusion and thermal expansion only, i.e.

$$
k_{2}=k_{\mathrm{d}}+k_{\mathrm{e}}
$$


where $k_{\mathrm{d}}$ and $k_{\mathrm{e}}$ are the constants for atom dissipation by diffusion and thermal expansion at any time, respectively; $K_{d}$ is defined $a^{28}$

$$
k_{\mathrm{d}}=k_{\mathrm{d}}^{0}\left(T / T_{0}\right)^{y}
$$

where $k_{\mathrm{a}}$ and $k_{\mathrm{d}}^{0}$ are diffusional rate constants at temperature $T$ and $T_{0}$, respectively; $y$ varies between 1.5 and $2.0,{ }^{28} K_{c}$ is given by ${ }^{29}$

$$
k_{\mathrm{e}}=(\mathrm{d} T / \mathrm{d} t) / T
$$

where $\mathrm{d} T / \mathrm{d} t$ is the heating rate at any temperature $T$.

For determining the temperature dependence of $k_{2}$, the values of $k_{\mathrm{d}}^{0}$ and $y$ should be obtained. For indium, the $y$ value of 1.84 can be found from Ref. 30. The value of $k_{\mathrm{d}}^{0}$ can be determined from the decay portion of the absorbance signal profile based on the method proposed by Akman et al. ${ }^{29}$

To determine $E_{\mathrm{a}}$ and $x$ based on equation (4), the terms of $\mathrm{d} A / \mathrm{d} t$ and $\int_{t}^{\infty} k_{2} A \mathrm{~d} t$ should be calculated. To this end, mathematical expressions of $A=f_{1}(t)$ and $k_{2} A=f_{2}(t)$ are obtained by polynomial regressions. Thus, the functions of $\mathrm{d} A / \mathrm{d} t=f^{\prime}{ }_{1}(t)$ and $\int_{t}^{\infty} k_{2} A$ $\mathrm{d} t=F_{2}(t)$ can be obtained by differentiating the expression of $A=f_{1}(t)$, and integrating $k_{2} A=f_{2}(t)$, respectively.

Data from the appearance to the maximum in the absorbance signal can be used to construct the plot based on equation (4) for determination of the kinetic order and activation energy since the amount of the analyte unatomized on the tube surface at time $t, P\left(\int_{t}^{\infty} k_{2} A \mathrm{~d} t-A\right)$, is greater than zero from the beginning at least to the maximum in the signal. In addition, special conditions to isolate the supply and removal function, and linear temperature ramp are indeed not required with the use of equation (4) because of no such assumptions made during the derivation of equation (4).

\section{EXPERIMENTAL}

\section{Apparatus}

A Perkin-Elmer model $\mathbf{4 0 0 0}$ atomic absorption spectrometer, equipped with a deuterium background corrector and a model HGA-400 graphite furnace was used for the measurement of the atomic absorption in the gas-stop mode. An indium hollow-cathode lamp (Shanghai Tienkuang Scientific Instruments Works, Shanghai, China) was employed at a lamp current of $5 \mathrm{~mA}$. The $303.9 \mathrm{~nm}$ line of indium was
Table 1. Furnace programme

\begin{tabular}{lrccr}
\hline & Dry & Ash & Atomize & Clean \\
\hline Ramp time (sec) & 20 & 5 & 1 & 1 \\
Hold time $(\mathrm{sec})$ & 50 & 30 & 5 & 2 \\
Temperature $\left({ }^{\circ} \mathrm{C}\right)$ & 130 & Various & Various & 2650 \\
Int. gas flow $(\mathrm{ml} / \mathrm{min})$ & 300 & 300 & 0 & 300 \\
\hline
\end{tabular}

used as the analysis line, with a spectral bandpass of $0.7 \mathrm{~nm}$. High purity argon gas was used as both sheath and purge gas. The furnace programme is given in Table 1 .

The graphite tube wall temperature was obtained from the output of a germanium phototransistor (B 1918-01, Hamamatsu), which was calibrated by focusing a disappearing filament optical pyrometer (Ircon Inc., UX-10, USA) through the sample injection hole in the graphite tube. The absorbance and temperature signal were synchronously acquired by a microcomputer-based data acquisition system at 20 -msec intervals. ${ }^{26}$ The signals could be subsequently stored on a diskette for further use.

Three different atomizer surfaces were used for the atomization of indium: Pyrolytically coated (PG), uncoated graphite tubes (UG) and the zirconium coated PG (ZrPG). The ZrPG were prepared according to Ref. 31 .

\section{Reagents}

The stock solution containing $1000 \mathrm{mg} / \mathrm{ml}$ In was prepared by dissolving $\operatorname{In}_{2} \mathrm{O}_{3}$ in an adequate volume of diluted nitric acid and subsequently diluting with deionized water. Working standards of the element were prepared daily from this stock solution with deionized water just before use. Indium working solutions were manually introduced into the furnace in $20-\mu 1$ volumes using an Eppendorf pipette.

A $5 \% \mathrm{~m} / \mathrm{v}$ of $\mathrm{ZrO}\left(\mathrm{NO}_{3}\right)_{2} \cdot 2 \mathrm{H}_{2} \mathrm{O}$ solution was prepared by dissolving $\mathrm{ZrO}\left(\mathrm{NO}_{3}\right)_{2} \cdot 2 \mathrm{H}_{2} \mathrm{O}$ in diluted nitric acid and then diluting with deionized water.

All chemicals were of analytical-reagent grade.

\section{RESULTS AND DISCUSSION}

Atomization from the pyrolytically coated graphite tube (PG)

The concentration study, which is illustrated in Fig. 1, contains absorbance-time plots for various initial masses of In deposited on the 


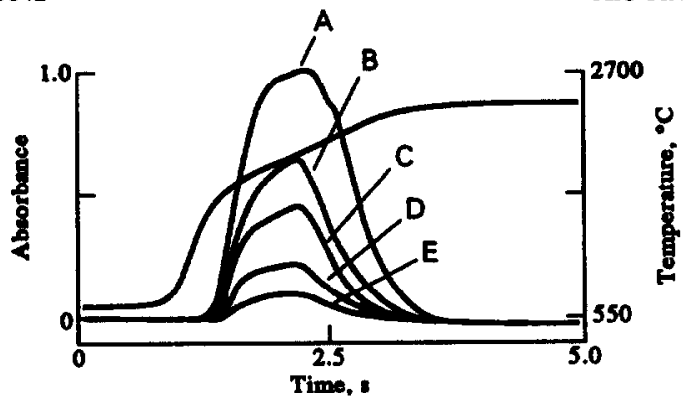

Fig. 1. Absorbance signal profiles for various masses of In from the pyrolytically coated graphite tube: (a) 5 (b) 3 (c) 2 (d) 1 (e) 0.5 ng. Ashing temperature: $500^{\circ} \mathrm{C}$, atomization temperature: $2600^{\circ} \mathrm{C}$, other conditions as listed in Table 1.

bottom of the PG. As the initial amount of In deposited on the surface of the PG is increased, peak times are similar and there is a slight shift to earlier appearance times. Thus, the apparent kinetic order for indium atomization from the PG appears to be unity. ${ }^{25}$ To obtain the kinetic order of indium atomization accurately, the plot of $\Delta \ln \left(\mathrm{d} A / \mathrm{d} t+k_{2} A\right) / \Delta \ln \left(\int_{i}^{\infty} k_{2} A \mathrm{~d} t-A\right) v s$ $\Delta(1 / T) / \Delta \ln \left(\int_{1}^{\infty} k_{2} A \mathrm{~d} t-A\right)$ is constructed from the absorbance signal profile. Figure 2 shows such a plot obtained from Fig. 1(c). As can be seen, the plot exhibits curvature, and so assignment of a single straight line from this plot is difficult, which may, in fact, reflect a continuous varying slope. This could imply gas-phase dissociation as step governing atom formation or a temperature dependence of activation energy $\left(E_{\mathrm{a}}\right)$ and kinetic order $(x)$ on surface coverage. Figure 3 shows the decomposition (curve $A$ ) and atomization (curve B) curves for indium from the PG. As can be seen, there is a gap between the temperature in the decomposition curve at which the absorbance begins to decrease (the maximum tolerant ashing temperature), and the appearance temperature in the atomization

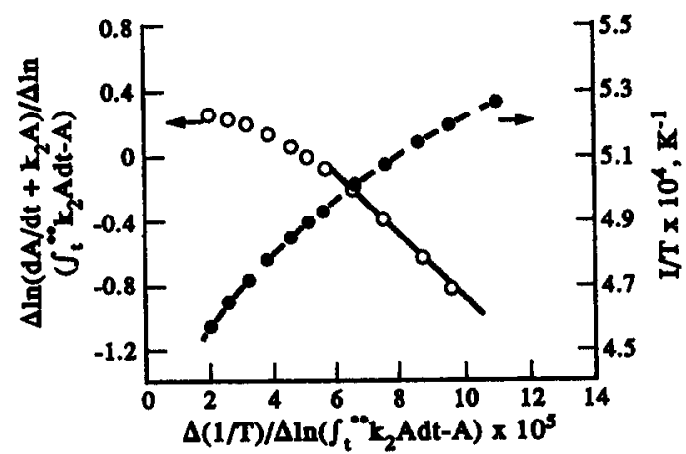

Fig. 2. Plot obtained from Fig. 1(c) based on equation (4) for $2 \mathrm{ng}$ of In from the pyrolytically coated graphite tube.

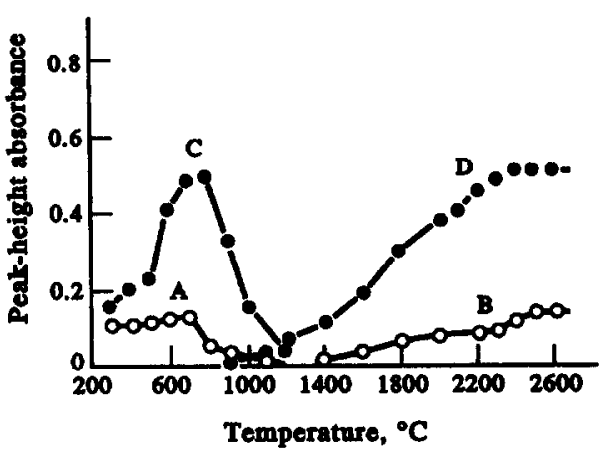

Fig. 3. Decomposition and atomization curves for $0.5 \mathrm{ng}$ of In by atomization from the pyrolytically coated graphite tube; (a) decomposition curve, at the atomization temperature of $2600^{\circ} \mathrm{C}$ (b) atomization curve, at the ashing temperature of $500^{\circ} \mathrm{C}$, and from the zirconium coated graphite tube; (c) decomposition curve, at the atomization temperature of $2400^{\circ} \mathrm{C}$ and (d) atomization curve, at the ashing temperature of $800^{\circ} \mathrm{C}$.

curve. This means that the decrease in the absorbance is not only due to loss of $\mathrm{In}_{(\mathrm{g})}$ but also to the formation of other gaseous species at temperatures above $700^{\circ} \mathrm{C}$. Calculations of thermodynamic equilibrium in the $\mathrm{In}-\mathrm{O}-\mathrm{C}$ system over the temperature range of $500-2000 \mathrm{~K}$ have shown that $\operatorname{In}_{2} \mathrm{O}_{(\mathrm{g})}$ is likely to be formed at either high ashing or lower atomization temperatures, but that $\mathrm{InO}_{(\mathrm{g})}$ should not play a significant role in the atomization of In. ${ }^{32}$ This has been confirmed by means of graphite furnace-mass spectrometry (GFMS) experiment. ${ }^{32}$ However, it is not easy to discuss the formation of free indium via gas-phase dissociation of an oxide in terms of kinetics since one must take into account the change in partial pressure of oxygen as well as the generation function of the indium oxide from the surface and the generation of the metal from the gas. ${ }^{33}$

An alternative explanation for the curvature observed in Fig. 2 may be that multiple release mechanisms contribute to the atomization of indium from the PG surface. If two straight lines are used to fit the plot in Fig. 2, then the activation energy $\left(E_{\mathrm{a}}\right)$ and kinetic order $(x)$ could be found to be 134 $\mathrm{kJ} / \mathrm{mol}$ and 0.97 at lower temperatures, 63 $\mathrm{kJ} / \mathrm{mol}$ and 0.37 at higher temperatures, respectively. This probably indicates that the ratelimiting step for the atomization of indium changes from a nearly first order kinetics with larger $E_{\mathrm{a}}$ value at lower temperatures into the kinetics of $x=0.37$ with lower $E_{\mathrm{a}}$ value at higher temperatures.

If we assume that either two straight lines or a continuous curve exists in Fig. 2 and that the 
intercept of any portion of the curve (i.e. the kinetic order $x$ ) would represent the state of the morphology of the analyte on the surface at that time, then one could conclude that the material moves from a dispersed state of $x=1$ to agglomerates (i.e. $x=0.37$ ). This suggests that the analyte begins as dispersed atoms or molecules containing indium and then forms droplets with increasing temperature. Although indium metal indeed exhibits weak interactions at the graphite observed by McNally and Holcombe, ${ }^{25}$ some indium molecular species may have a strong interaction which causes it to be dispersed on the surface and exhibited nearly first order release at lower temperatures. The obtained larger $E_{\mathrm{a}}$ value $(134 \mathrm{~kJ} / \mathrm{mol})$ could represent indium molecular species-graphite interaction energy. Furthermore, the weak indium metal-graphite interactions would foster the formation of droplets which is consistent with the higher temperature order of release $(x=0.37)$. The observed lower $E_{\text {a }}$ value may represent the energy required for indium release at the edge of the metal-graphite interface. ${ }^{25,34}$ It is possible that indium metal would be formed either through carbon reduction of the oxide or the thermal decomposition of the oxide at higher temperatures. In addition, no significant influence of the initial injected mass of indium studied on the indium atomization from the PG is observed, as shown in Table 2.

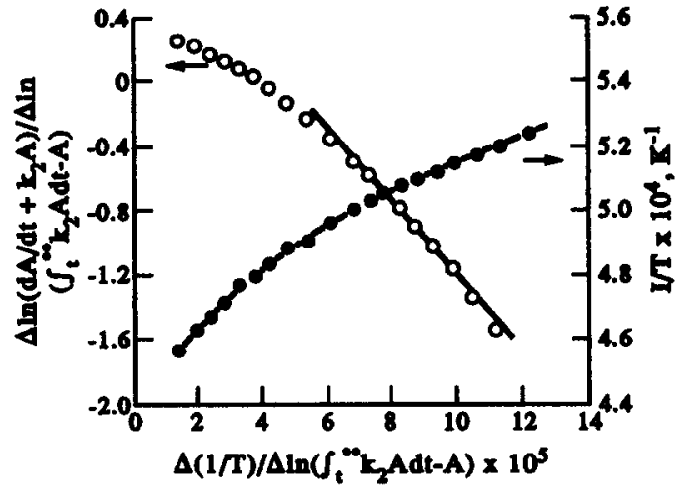

Fig. 4. Plot constructed based on equation (4) for $2 \mathrm{ng}$ of In from the uncoated graphite tube. Other conditions as in Table 1.

\section{Atomization from the uncoated graphite tube (UG)}

In order to examine surface spreading and penetration by the aqueous solution of the sample on the atomization of indium, the kinetic parameters of indium atomization from the uncoated graphite tube (UG) have been determined. Figure 4 illustrates the plot of $\Delta \ln \left(\mathrm{d} A / \mathrm{d} t+\mathrm{k}_{2} \mathrm{~A}\right) / \Delta \ln \left(\int_{t}^{\infty} k_{2} A \mathrm{~d} t-A\right) v s$ $\Delta(1 / T) \Delta \ln \left(\int_{t}^{\infty} k_{2} A \mathrm{~d} t-A\right)$ from the absorbance signal profile for $2 \mathrm{ng}$ of indium ashed at $500^{\circ} \mathrm{C}$ and atomized at $2600^{\circ} \mathrm{C}$ from the UG. Compared with Fig. 2, similar results can be obtained from Fig. 4, i.e. Fig. 4 also exhibits nonlinearity, reflecting a continuous varying slope and intercept. If two straight lines are

Table 2. Kinetic parameters for indium atomization from different atomizer surfaces ( \pm standard deviation of three determinations)

\begin{tabular}{clcc}
\hline Tube & Mass of In $(\mathrm{ng})$ & Kinetic order & $E_{\mathrm{a}}(\mathrm{kJ} / \mathrm{mol})$ \\
\hline PG & 0.5 & $0.35 \pm 0.05 / 0.97 \pm 0.05$ & $50 \pm 13 / 138 \pm 13$ \\
& 1 & $0.36 \pm 0.03 / 0.96 \pm 0.03$ & $51 \pm 12 / 142 \pm 13$ \\
& 2 & $0.40 \pm 0.04 / 0.99 \pm 0.06$ & $63 \pm 10 / 146 \pm 13$ \\
& 3 & $0.37 \pm 0.01 / 0.98 \pm 0.07$ & $50 \pm 4 / 138 \pm 15$ \\
& 5 & $0.37 \pm 0.04 / 1.02 \pm 0.06$ & $42 \pm 4 / 126 \pm 17$ \\
& Av. & $0.37 \pm 0.02 / 0.98 \pm 0.02$ & $51 \pm 7 / 138 \pm 8$ \\
UG & 0.5 & $0.38 \pm 0.02 / 0.95 \pm 0.06$ & $56 \pm 8 / 142 \pm 17$ \\
& 1 & $0.34 \pm 0.04 / 0.98 \pm 0.04$ & $50 \pm 10 / 126 \pm 13$ \\
& 2 & $0.36 \pm 0.06 / 0.94 \pm 0.07$ & $54 \pm 15 / 138 \pm 17$ \\
& 3 & $0.37 \pm 0.02 / 1.04 \pm 0.05$ & $50 \pm 6 / 134 \pm 13$ \\
& 5 & $0.35 \pm 0.04 / 1.02 \pm 0.06$ & $59 \pm 13 / 126 \pm 8$ \\
& Av. & $0.36 \pm 0.02 / 0.98 \pm 0.04$ & $54 \pm 4 / 133 \pm 8$ \\
PG + Pd 0.25 & $1.04 \pm 0.06$ & $406 \pm 25$ \\
& 0.5 & $1.02 \pm 0.07$ & $460 \pm 25$ \\
& 0.75 & $1.08 \pm 0.11$ & $414 \pm 8$ \\
& 1 & $1.08 \pm 0.09$ & $402 \pm 20$ \\
& Av. & $1.06 \pm 0.03$ & $421 \pm 27$ \\
ZrPG & 0.25 & $0.61 \pm 0.07$ & $204 \pm 6$ \\
& 0.5 & $0.69 \pm 0.05$ & $185 \pm 11$ \\
& 0.7 & $0.59 \pm 0.04$ & $174 \pm 10$ \\
& 0.9 & $0.68 \pm 0.09$ & $180 \pm 13$ \\
Av. & $0.64 \pm 0.05$ & $186 \pm 13$ \\
\hline
\end{tabular}


used to fit Fig. 4, the kinetic order and activation energy can be found to be 0.97 and $155 \mathrm{~kJ} / \mathrm{mol}$, at lower temperatures, respectively; while at higher temperatures, the kinetic order of 0.38 and activation energy of $67 \mathrm{~kJ} / \mathrm{mol}$ can be obtained. Although the uncoated graphite surface is expected to show much more porous than the pyrolytically coated graphite surface, the near identity of the kinetic parameters indicates that the atomization mechanisms of indium are similar for both atomizer surfaces.

\section{Atomization from the $P G$ surface in the presence of palladium}

It has been shown that palladium is an effective matrix modifier for the determination of indium, which greatly improves the sensitivity and raises the maximum tolerant ashing temperature. ${ }^{35}$ In this work, the effect of the presence of palladium on the atomization mechanism of indium is investigated. The palladium modifier is pretreated before each determination by injecting a $20 \mu \mathrm{l}$ of $100 \mu \mathrm{g} / \mathrm{ml}$ Pd into the PG through the sample injection hole, drying at $150^{\circ} \mathrm{C}$ for $30 \mathrm{~s}$ and heating at $900^{\circ} \mathrm{C}$ for $30 \mathrm{sec}$. Figure 5 shows the plot of $\Delta \ln \left(\mathrm{d} A / \mathrm{d} t+k_{2} A\right) / \Delta \ln \left(\int_{t}^{\infty} k_{2} A \mathrm{~d} t-A\right)$ os $\Delta(1 / T) / \Delta \ln \left(\int_{t}^{\infty} k_{2} A \mathrm{~d} t-A\right)$ from the absorbance signal profile for $0.5 \mathrm{ng}$ of In ashed at $500^{\circ} \mathrm{C}$ and atomized at $2600^{\circ} \mathrm{C}$ from the PG surface in the presence of palladium. In contrast to Figs 2 and 4, a single straight line can be fit well to the plot shown in Fig. 5. This means that a single mechanism is active in the atomization of indium with palladium. From Fig. 5 the activation energy and kinetic order are found to be $414 \mathrm{~kJ} / \mathrm{mol}$ and 0.95 , respectively. No significant influence of the initial injected mass of indium studied on the kinetics of indium atomization is observed, as shown in

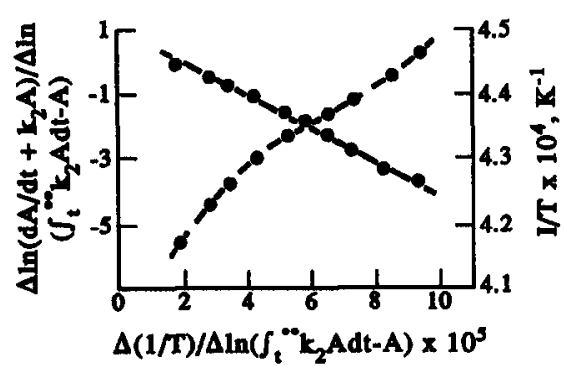

Fig. 5. Plot obtained by using equation (4) for $0.5 \mathrm{ng}$ of In in the presence of $2 \mu \mathrm{g}$ of Pd from the pyrolytically coated graphite tube. Other conditions as in Fig. 1.

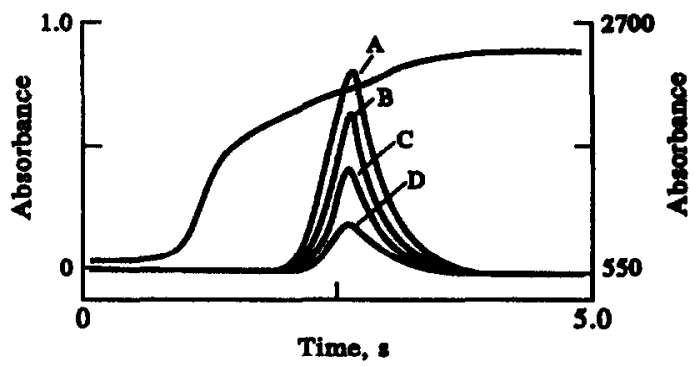

Fig. 6. Absorbance signal profiles for various masses of In from the pyrolytically coated graphite tube when $2 \mu \mathrm{g}$ of Pd is used as a modifier: (a) 1 ; (b) 0.75 ; (c) 0.5 ; (d) $0.25 \mathrm{ng}$. Other conditions as in Fig. 1.

Table 2. The first order kinetics can also be seen from the concentration study depicted in Fig. 6. These results suggest that the atomization mechanism of indium in the presence of palladium is different from those for the PG and UG in the absence of palladium.

Figure 7 shows the absorbance signal profiles for $0.5 \mathrm{ng}$ of $\mathrm{In}$ ashed at $500^{\circ} \mathrm{C}$ and atomized at $2600^{\circ} \mathrm{C}$ from the PG with (curve $A$ ) and without (curve B) the palladium modifier. Obviously, in the presence of palladium the appearance of the absorbance signal is significantly delayed, and both the peak height and peak area are increased. The increase in the sensitivity indicates that the use of a palladium modifier prevents the formation of gaseous species in the presence of palladium. By using Fourier transform infrared spectrometry, Volynsky et al. ${ }^{36}$ found that the palladium modifier sharply decreased the reduction temperature of the metal oxides with graphite. It was regarded that the catalytic property of palladium facilitated the carbon reduction of oxides at low ashing temperatures. ${ }^{36}$ Thus, we assume that in the presence of palladium the oxide $\operatorname{In}_{2} \mathrm{O}_{3(\mathrm{~s})}$ is rapidly reduced to metallic indium, and the resultant indium metal forms the intermetallic compounds or solid solution ${ }^{37.38}$ with the

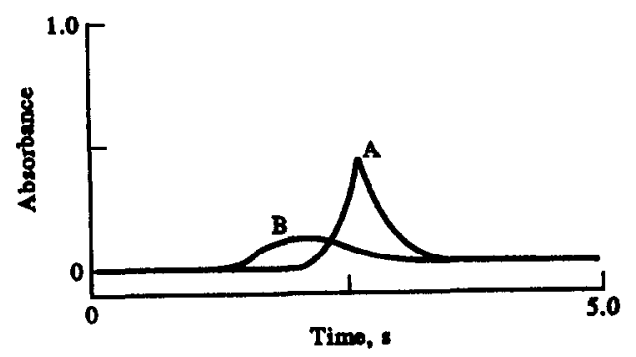

Fig. 7. Effect of palladium modifier on the absorbance signal profile of indium from the pyrolytically coated graphite tube: (a) $0.5 \mathrm{ng}$ In $+2 \mu \mathrm{g}$ Pd; (b) $0.5 \mathrm{ng}$ In. Conditions as shown in Fig. 1. 
palladium metal, thus decreasing or almost eliminating loss of the analyte due to the formation of gaseous oxide. At the atomization stage, the absorbance is produced owing to the release of indium from the solid solution with Pd, which may cause the observed first order kinetics. The obtained $E_{\mathrm{a}}$ value $(421 \pm 27 \mathrm{~kJ} / \mathrm{mol})$ represents the energy required to release the indium in molten palladium, which depends on the heat of solution of the In and the pd.

Atomization from the zirconium coated graphite tube ( $\mathrm{ZrPG}$ )

The absorbance signal profiles for different amounts of indium atomized from the zirconium coated graphite tube ( $\mathrm{ZrPG}$ ) under optimum conditions are depicted in Fig. 8, from which the fractional kinetic order for the atomization of indium from the ZrPG can be seen since the peak maximum occurs later with increasing analyte mass. ${ }^{34}$ In order to obtain the accurate value of the kinetic order, plots of $\Delta \ln \left(\mathrm{d} A / d t+k_{2} A\right) / \Delta \ln \left(\int_{1}^{\infty} k_{2} A d t-A\right) v s$ $\Delta(1 / T) / \Delta \ln \left(\int_{t}^{\infty} k_{2} A d t-A\right)$ are constructed. Figure 9 shows such a plot from Fig. 8(d). The obtained values of the kinetic order and activation energy from Fig. 9 are 0.64 and 192 $\mathrm{kJ} / \mathrm{mol}$, respectively. There is no remarkable change in the kinetic parameters for indium atomization from the ZrPG with the initial injected amount of In studied, as can be seen from Table 2. The above results imply that the atomization mechanisms of indium are different for the $\mathrm{ZrPG}$ and PG.

Figure 3 also shows the decomposition (curve C) and atomization (curve D) curves for indium from the $\mathrm{ZrPG}$. As can be seen, there is no significant gap between the maximum tolerant ashing temperature and the appearance

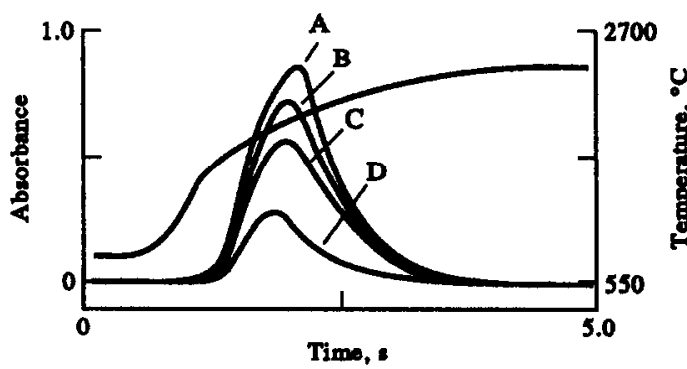

Fig. 8. Absorbance signals for various amounts of In from the zirconium coated graphite tube: (a) 0.9 (b) 0.7 (c) 0.5 (d) $0.25 \mathrm{ng}$. Ashing temperature: $800^{\circ} \mathrm{C}$, atomization temperature: $2400^{\circ} \mathrm{C}$.

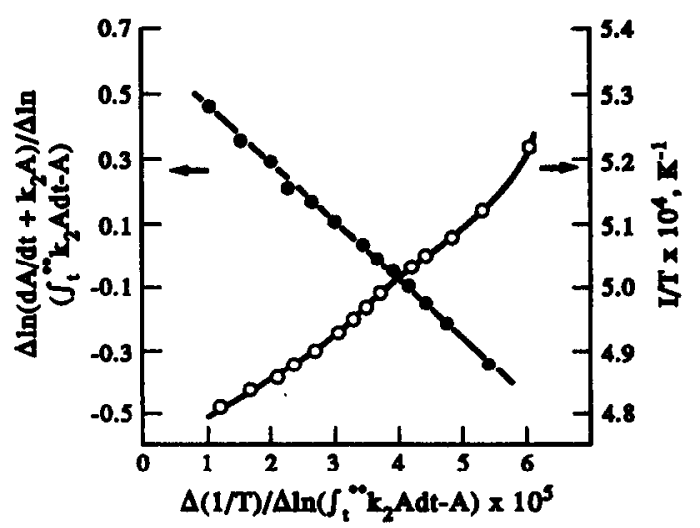

Fig. 9. Plot obtained from Fig. 8(d) by using equation (4) for $0.25 \mathrm{ng}$ of In from the zirconium coated graphite tube.

temperature in the atomization curve. Thus, compared with the PG (curves A and B in Fig. 3), the ZrPG seems to prevent the formation of gaseous species and greatly improves the sensitivity. It has been suggested that prevention of oxide formation may be the main reason for the better sensitivity of determinations on the refractory element coated furnace. ${ }^{39}$ Probably more active reducing properties of refractory element carbides than graphite are responsible for the prevention of gaseous oxide formation. ${ }^{20.40}$ Increase in the absorbance with ashing temperature below $700^{\circ} \mathrm{C}$ (curve $\mathrm{C}$ in Fig. 3) indicates that the reducing activity of $\mathrm{ZrC}$ may increase with temperature.

On the basis of the above, the following reactions are supposed for indium atomization from the $\mathrm{ZrPG}$, similar to those discussed in Refs 20, 41;

$$
\begin{aligned}
\mathrm{ZrC}_{(\mathrm{s})}+\mathrm{In}_{2} \mathrm{O}_{3(\mathrm{~s})} & \rightarrow \mathrm{Zr}_{(\mathrm{s})}+\mathrm{In}_{(\mathrm{s} / 1)}+\mathrm{CO}_{(\mathrm{g})} \\
\mathrm{ZrC}_{(\mathrm{s})}+\mathrm{In}_{2} \mathrm{O}_{(\mathrm{g})} & \rightarrow \mathrm{Zr}_{(\mathrm{s})}+\mathrm{In}_{(\mathrm{s} / 1)}+\mathrm{CO}_{(\mathrm{g})} \\
\mathrm{In}_{(\mathrm{s} / 1)} & \rightarrow \mathrm{In}_{(\mathrm{g})}
\end{aligned}
$$

The resulting metallic zirconium reacts with graphite to form $\mathrm{ZrC}$ again at high temperatures, while the formed indium metal vaporizes with increasing temperature. The obtained kinetic order of near $2 / 3$, along with the activation energy of $192 \mathrm{~kJ} / \mathrm{mol}$, indicates that the atomization of indium may be governed by the surface area of the sphere of molten metal, and the interaction between indium metal and zirconium carbide surface is relatively weak.

\section{CONCLUSION}

The kinetics of indium atomization seems to be similar for the pyrolytically coated and the 
uncoated graphite tubes, but different for the zirconium coated graphite tube. The use of a palladium modifier or of the zirconium coated tube changes the path of indium atomization, inhibiting the formation of gaseous indium oxide, and hence greatly improves the sensitivity for indium determination.

Acknowledgement-This work was supported by the Chinese Academy of Sciences under grant No. KM 85-47.

\section{REFERENCES}

1. C. W. Fuller, Analyst, 1974, 99, 739.

2. G. Tessari and G. Torsi, Anal. Chem., 1975, 47, 842.

3. G. Torsi and G. Tessari, Anal. Chem., 1975, 47, 839.

4. B. V. L'vov, P. A. Bayunov and G. N. Ryabchuk, Spectrochim. Acta, 1981, 36B, 397.

5. D. Rojas and W. Olivares, Spectrochim. Acta, 1992, 47B, 387.

6. W. C. Campbell and J. M. Ottaway, Talanta, 1974, 21, 837.

7. B. V. L'vov and A. S. Savin, Zh. Anal. Khim., 1983, 38, 1925.

8. J. P. Byyrne, Aust. J. Chem., 1979, 32, 249.

9. W. French, E. Lundberg and A. Cedergren, Prog. Anal. Atom. Spectrosc., 1985, 8, 257.

10. C. L. Chakrabarti, S. B. Chang and S. E. Roy, Spectrochim Acta, 1983, 38B, 447.

11. R. E. Sturgeon, C. L. Chakrabarti and C. H. Langford, Anal. Chem., 1976, 48, 1792.

12. O. Genc, S. Akman, A. R. Ozdural, S. Ates and T. Balkis, Spectrochim. Acta, 1981, 36B, 163

13. B. Smets, Spectrochim. Acta, 1980, 35B, 33.

14. M. Suzuki, K. Ohta and K. Isobe, Anal. Chim. Acta, 1985, 173, 321.

15. M. Suzuki and K. Ohta, Anal. Chim. Acta, 1983, 151, 401 .

16. J. Aggett and A. J. Sprott, Anal. Chim. Acta, 1974, 72, 49.

17. D. C. Gregoire and C. L. Chakrabarti, Spectrochim. Acta, 1982, 37B, 611 .
18. M. Suzuki, K. Ohta, T. Yamakita and T. Katssuno, Spectrochim. Acta, 1981, 36B, 679.

19. O. Vyskocilova, V. Sychra and D. Kolihova, Anal. Chim. Acta, 1979, 105, 271.

20. Y.-Q. Gao and Z.-M. Ni, Acta Chim. Sin., 1982, 40, 1021 .

21. C. L. Chakrabarti and S. J. Cathum, Talanta, 1990, 37, 1111

22. R. W. Fonseca, O. A. Guell and J. A. Holcombe, Spectrochim. Acta, 1990, 45B, 1257.

23. I. L. Grinshtein, L. A. Vasil'eva and D. A. Katskov, $Z h$. Prikl. Spectrask., 1985, 46, 13.

24. D. A. Katskov, I. L. Grinshtein and L. P. Kriglikova, Zh. Prikl. Spectrosk., 1980, 33, 804.

25. J. McNally and J A. Holcombe, Anal. Chem. 1991, 63, 1918

26. X.-P. Yan, Z.-M. Ni, X.-T. Yang and G.-Q. Hong, Spectrochim. Acta, Part B, in press.

27. X.-P. Yan and Z.-M. Ni, Proc. of Int. Fourth Beijing Conference and Exhibition on Instrumental Analysis, 1991, Beijing, Abs. C70.

28. B. V. L'vov, Spectrochim. Acta, 1961, 17B, 701.

29. S. Akman, S. Bektas and O. Genc, Spectrochim. Acta, $1990,43 B, 763$

30. B. V. L'vov, Spectrochim. Acta, 1990, 45B, 633.

31. X.-P. Yan and Z. M. Ni, J. Anal. At. Spectrom., 1991, $6,483$.

32. T. McAllister, J. Anal. At. Spectrom., 1990, 5, 171.

33. J. A. Holcombe, Spectrochim. Acta, 1989, 448, 975.

34. J. McNally and J. A. Holcombe, Anal. Chem., 1987, 59, 1105.

35. Z.-M. Ni and X.-Q. Shan, Spectrochim. Acta, 1987, 42B, 937.

36. A. Volynsky, S. Tikhomirov and A. Elagin, Analyst, 1991, 116, 145.

37. A. F. Trotman-Dickenson, Comprehensive Inorganic Chemistry, Vol. 1. Pergamon Press, Oxford, 1973.

38. P.-Y. Yang, Z.-M. Ni, Z.-X. Zhuang, F.-C. Xu and A.-B. Jiang, J. Anal. At. Spectrom., 1992, 7, 515.

39. A. B. Volynsky, E. M. Sedykh and B. Ya Spivakov, Anal. Chim. Acta, 1985, 174, 173.

40. G. Muller-Vogt and W. Wendll, Anal. Chem., 1981, 53, 651 .

41. W. B. Blumenthal, The Chemical Behavior of Zirconium, Princeton, N.J., D. Van Nostrad, New York, 1958. 